NISTIR 6896

2002

\title{
UTILITY OF HIGH PRESSURE OIL WITH DIGITAL PROPORTIONAL VALVES IN SERVO APPLICATIONS
}

\author{
for \\ Naval Surface Warfare Center \\ Carderock Division \\ 9500 MacArthur Blvd. \\ West Bethesda, MD 20817-5700
}

Richard J. Norcross

Intelligent Systems Division

National Institute of Standards and Technology

Gaithersburg, MD 20899-8230 


\section{Disclaimer}

No approval or endorsement of any commercial product by the National Institute of Standards and Technology is intended or implied. Certain commercial equipment, instruments, or materials are identified in this report to facilitate understanding. Such identification does not imply a recommendation or endorsement by the National Institute of Standards and Technology, nor does it imply that the materials or equipment identified are necessarily the best available for the purpose.

\section{Copyright}

This publication was prepared by United States Government employees as part of their official duties and is, therefore, a work of the U.S. Government and not subject to copyright.

\section{Acknowledgement}

This report is partial fulfillment of sub-contract AM 02-9802001 with AmDyne Corporation of Millersville, Maryland. 


\section{Abstract}

The Carderock Division of the Naval Surface Warfare Center is developing the Automated Paint Application, Containment, and Treatment System (APACTS). APACTS will apply anti-corrosive and anti-fouling paints to ship hulls in an environmentally sound manner. To provide accurate motion over very large surfaces, the APACTS motion system employs a self-propelled base carrying a long reach macro-manipulator, which in turn carries a quick response micromanipulator to maneuver the paint nozzle and containment device. The macro-manipulator is a commercial shooting boom aerial work platform (AWP). The AWP uses digital proportional hydraulic valves to control the oil flow to the actuators. The APACTS control system coordinates the flow to cause the macromanipulator to move the micromanipulator along the ship's contours. The APACTS developer desires to operate the AWP's hydraulic system at higher then normal pressures. The added pressure generates the speeds and accelerations required by APACTS micro-manipulator to maintain nozzle trajectory relative to the surface. The experiments reported here indicate the servo performance of the standard digital proportional valves is retained with the increased oil feed pressures. 


\section{Table of Contents}

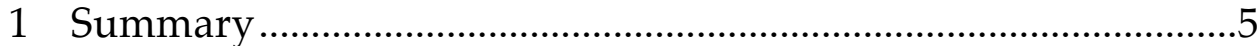

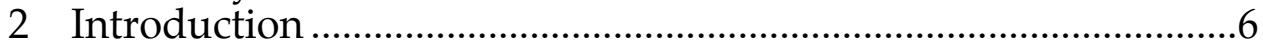

3 Methods, Assumptions, and Procedure........................................7

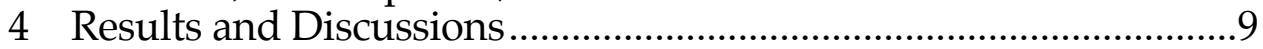

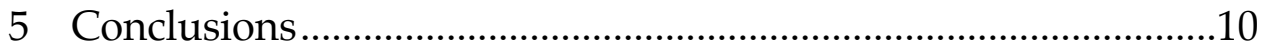

6 Recommendations .................................................................. 10

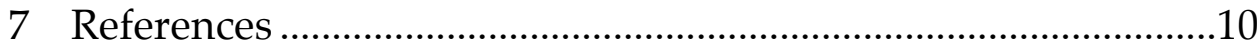

\section{List of Figures}

Figure 1. APACTS System Concept .....................................................5

Figure 2 Lift Actuator Response to Oil Feed Pressures......................9 


\section{Summary}

The Carderock Division of the Naval Surface Warfare Center is developing the Automated Paint Application, Containment, and Treatment System (APACTS) to apply anti-corrosive and anti-fouling paints onto Navy ship hulls in an environmentally sound manner. APACTS (Figure 1) employs three motion components: (1) a self-propelled base, (2) a long reach macro-manipulator, and (3) a quick response micromanipulator to move the paint nozzle and containment device along the hull surface. The combined motion trajectories of the macro- and micromanipulators produce the paint application trajectory. Feedback from acoustic sensors mounted on the containment device modify the trajectories in real-time to keep the nozzle along an appropriate trajectory relative to the surface.

\section{Figure 1. APACTS System Concept}

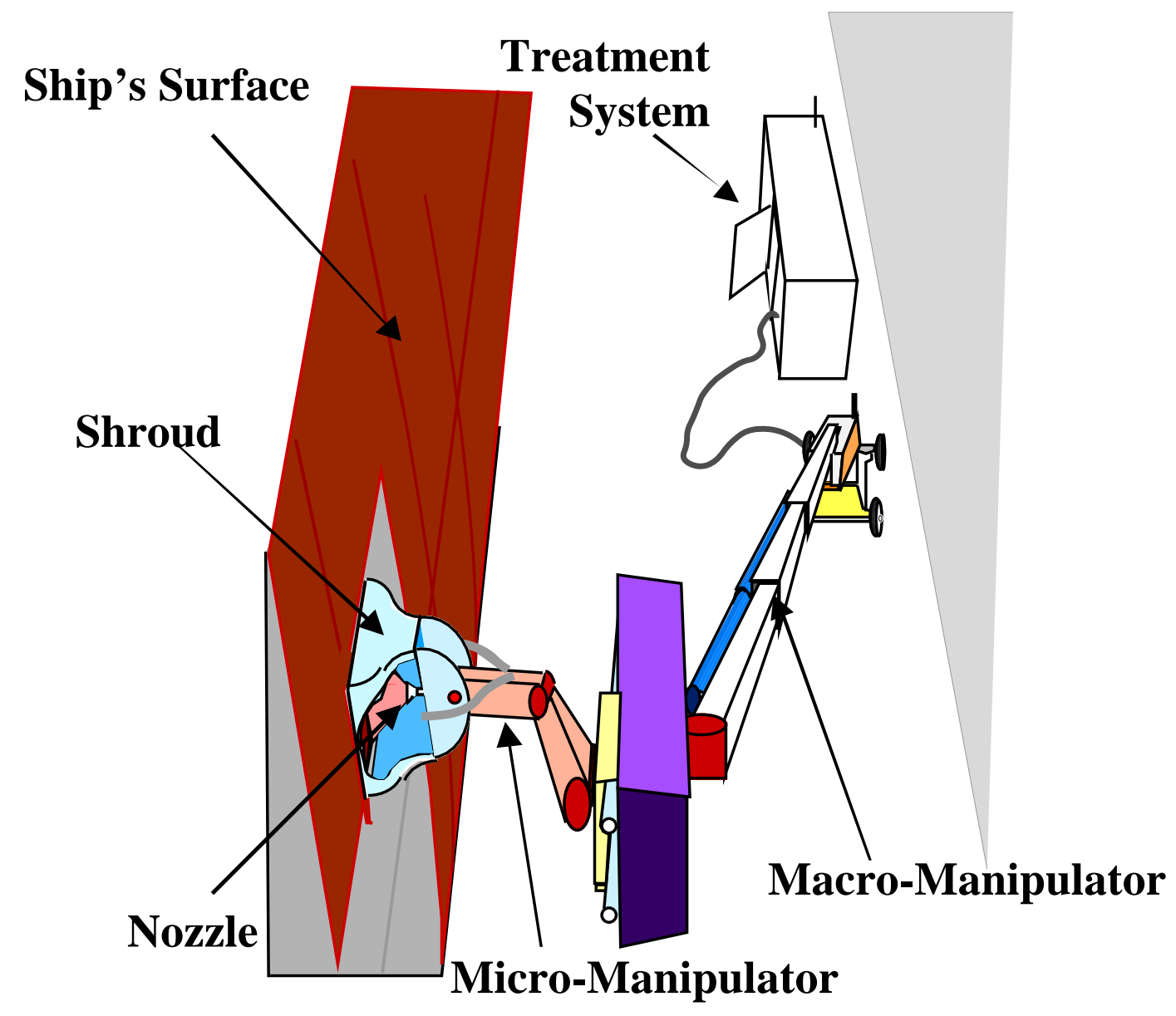

Utility of High Pressure oil with Digital Proportional Valves in Servo Applications 
The controller follows the trajectory by making small changes to the positions of the AWP's actuators. A servo controller compares the current actuator position with the desired position and adjusts a signal to the actuator's valve. The valve directs oil from a high pressure feed line, to the actuator, and back to a low pressure tank line. The actuator motion is a function of the oil flow.

Previous APACTS research developed servo controlled actuators for the macromanipulator. That system used the standard hydraulic system provided by the manufacturer. Those hydraulics generated $12 \mathrm{MPa}$ oil feed pressure. The system produced stable motion at low velocities and accelerations.

The APACTS micro-manipulator will use higher feed pressures (>17 $\mathrm{MPa})$ to generate the higher speeds and accelerations required to maintain the nozzle trajectory. While the standard components are rated above $17 \mathrm{MPa}$ the effects of the increased feed pressure on the macro-manipulator's performance are unknown. In all situations, only a portion of the valve input range produces controllable motions. The remainder causes either no motion or the maximum velocity. The increased feed pressure could cause the effective portion of the valve input to become too small for meaningful control.

These experiments compare open loop actuator velocities with feed pressures. The results indicate that the actuator's maximum velocity is reduced when the feed pressure to a digital proportional valve is increased to $17 \mathrm{MPa}$. However, the ratio between the effective input range and the velocity range remains similar. Therefore the aerial work platform's standard digital proportional valves should produce adequate servo control when operated with feed pressures around $17 \mathrm{MPa}$.

\section{Introduction}

To guard against the harshness of the sea, ships are covered with anti-corrosive and anti-fouling paints that must be periodically replaced to maintain their effectiveness. During replacement, hazardous airborne particles (HAPs) are inadvertently discharged into the environment diminishing the air quality and the surrounding harbor waters. The Naval Sea Systems Command, Naval Surface Warfare Center, Carderock Division (NAVSEA NSWCCD), Environmental Quality Department conducts research and development leading to fleet implementation of pollution-control materials, processes, and equipment that enable Navy ships to be environmentally responsible. NSWCCD is responsible for providing the Navy with the technical expertise to solve existing and emerging waste management problems. Pursuant to that responsibility, NSWCCD is developing the Automated Paint Application, Containment, and Treatment System (APACTS) to significantly reduce HAP discharge from the painting operation [1].

The primary components of APACTS are the delivery, containment, treatment, and manipulation systems. The delivery system consists of a paint mixer, 
strainer, sprayer, nozzle, and associated equipment. The containment system surrounds the paint sprayer and includes a capture shroud, recovery vacuum, hoses, and controls. The treatment system includes waste transport, waste isolation, filter elements, and their support equipment. The manipulation system consists of those devices that move the sprayer and containment shroud. The components complement each other to produce an effective, economic, and environmentally sound system.

The Intelligent Systems Division of the National Institute of Standards and Technology (NIST-ISD) supports APACTS development through the investigation of new and existing technologies to carry, maneuver, and manipulate the APACTS sprayer and containment system. Because a single manipulator would be unable to achieve all of the Navy's performance requirements at an acceptable cost, APACTS uses a series of three manipulators to position the system about the dry dock, to reach along the hull, and to maintain proper standoff and motion. After the mobile base positions APACTS in or around the drydock, a long reach but slow response macro-manipulator carries a high accuracy, fast response micro-manipulator to simultaneously provide sufficient reach and accuracy.

Commercial AWP manufacturers use digital proportional valves to provide low cost, variable speed control to their machines. The valves have significantly lower dynamic response than traditional hydraulic servo valves. In addition the valves display a significant deadband around zero velocity. The valve system includes a response feedback signal to the hydraulic pump that adds hysteresis. In general, the valves are inadequate for servo control.

However, the valves support servo control when operated under certain conditions. An extra feedback line generates constant feed pressure of $12 \mathrm{MPa}$ $\left(1750 \mathrm{lb} / \mathrm{in}^{2}\right)$ and removes most of the valve system hysteresis. The valve's dynamic response is sufficient when trajectories are limited to slowly changing, non-zero velocities. Implementation with these strategies and limitations produces satisfactory servo control of a commercial AWP's actuators and coordinated motion.

The APACTS developers intend to use the AWP's hydraulic pump to also power the micromanipulator. The micromanipulator requires a much higher feed pressure to generate that manipulator's desired dynamic response. The developers fear that the higher pressure will eliminate the valve's ability to generate unique actuator velocities. This paper reports on experiments to assess the AWP valve's ability to perform with higher feed pressures.

\section{Methods, Assumptions, and Procedure}

The performance test consists of a series of open loop velocity comparisons. The open loop velocity is the actuator's steady state speed for a constant input signal. For linear servo control, the actuator's speed must be proportional to the input 
signal. Furthermore, the servo system requires a range of possible outputs. The servo controller cannot generate smooth motion if the slope between the input signal and output speed is too steep.

The AWP's valves use a $30.77 \mathrm{~Hz}$ Pulse Width Modulated signal to command oil flow. The controller divides each $32.5 \mathrm{~ms}$ pulse into 1040 equal segments of 31.25 $\mu$ s. During a pulse, the valve is "on" for the first set of segments and "off" for the remainder. The valve's spool has $2.7 \mathrm{MPa}$ (400 psi) oil reservoirs at both ends. When the signal is on, the oil drains from one end of the spool. The higher pressure of the opposing reservoir drives the spool off center. When the signal is off, the reservoir pressure returns and the spool centers with the aid of springs. The longer the "on" segment, the further the spool moves off of the closed position. The ratio between the number of on segments to the number of segments per pulse is the Duty Cycle. With sufficient Duty Cycle, oil flows through the valve to the actuator causing motion.

The actuator must move in proportion to the Duty Cycle. In order to have servo control, the output must be proportional to the input. If an increased input results in an increased output sometimes and a decreased output other times, a linear servo controller cannot control the position of the actuator.

In these experiments, the controller issues an open-loop command and monitors the actuator position. After several seconds, the experimenter commands the actuator to stop. During the movement, the controller records the actuator position at regular intervals. At the end of the run, the controller performs a position versus time linear reduction on the later $90 \%$ of values. The slope of this reduction is the steady state velocity for the specific Duty Cycle.

The experimenter collects steady state velocities throughout the operating range of the valve. The valve's closed center prevents actuator movement for much of the command input. The experiment compares the inputs for which there is motion.

The Snorkel hydraulic system uses a variable displacement pump. A feedback sense line and a pressure regulator control the pump's output pressure. In normal operation, the sense line connects the actuators with the control plate of the hydraulic pump and the pump maintains a feed pressure that is greater then the pressure being used by the actuators. For servo control, a constant feed pressure helps stabilize the valve output. Thus an extra valve (one without an actuator) provides a phantom feedback pressure. For these experiments, the sense line connects directly to the pump output and the pressure regulator directly controls the system's hydraulic pressure.

The experimenter collects sets of steady state velocities for various feed pressures and plots command Duty Cycle versus actuator speed. If the output results at higher pressures follow the commands similar to the results for $12 \mathrm{MPa}$ $\left(1750 \mathrm{lb} / \mathrm{in}^{2}\right)$, then APACTS can establish servo control at the higher pressures. 


\section{Results and Discussions}

The plot in Figure 2 shows the output response to the command Duty Cycle for system oil pressures between $10 \mathrm{MPa}$ and $17 \mathrm{MPa}\left(1500 \mathrm{lb} / \mathrm{in}^{2}\right.$ and $\left.2500 \mathrm{lb} / \mathrm{in}^{2}\right)$. The figure shows two curves for $14 \mathrm{MPa}\left(2200 \mathrm{lb} / \mathrm{in}^{2}\right)$. In this region, a dramatic variation in the response occurred. In other regions, the responses of the repeat runs were similar and only one is shown.

Figure 2 Lift Actuator Response to Oil Feed Pressures

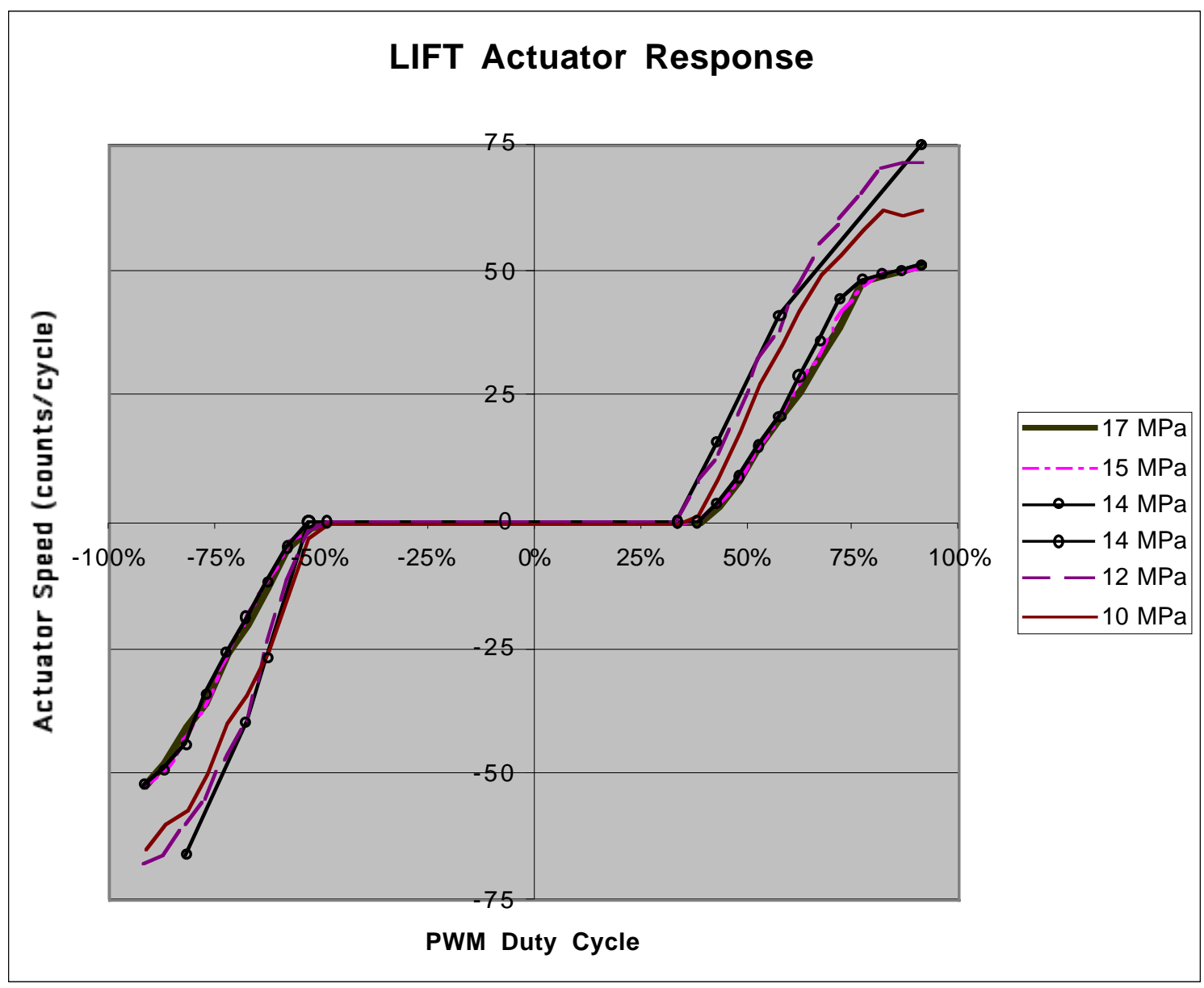

The standard valve on a commercial aerial work platform is not a perfectly linear control device. When the system feed pressure increases from $10 \mathrm{MPa}$, the actuator's response initially becomes steeper with an accompanying increase to the maximum speed and reduction to the command resolution. Around $14 \mathrm{MPa}$, the system's response becomes unpredictable. Above $15 \mathrm{MPa}$, the system again displays proportional response, although with a smaller maximum velocity. 


\section{Conclusions}

A comercial aerial work platform has hydraulic components designed for $20 \mathrm{MPa}$ oil pressure. However the manufacturer restricts the pump output for normal operations. The APACTS designers intend to increase the pump output to provide sufficient pressure for the micro-manipulator to achieve required dynamic response. The increased pressure's effect on the performance of the macro-manipulator is the subject of this investigation.

The standard valves provided with the commercial aerial work platform will perform adequately when the feed pressure is maintained above $15 \mathrm{MPa}(2300$ $\left.\mathrm{lb} / \mathrm{in}^{2}\right)$. The slope of the output versus input is similar at the higher pressures as with the currently utilized operating pressure. However, the maximum actuator velocity will be less then with the current coordinated control system. This reduction will still allow the APACTS to function in the environment in which it is intended.

The researchers suppose the speed limitation at higher feed pressures is an effect of the constant spool drive pressure. The valves maintain 2.7 MPa pilot oil to move the spools. The higher oil pressure in the valve's pressure cavity may provide increased resistance via the spool seals. The anomaly observed at 14 $\mathrm{MPa}$ would be the point when this factor first takes effect.

\section{Recommendations}

The experimenters did not investigate the anomaly observed at $14 \mathrm{MPa}$ nor the maximum speed reduction. The speed reduction will not be a factor in the APACTS application. The anomaly effect was highly repeatable but safely outside the range of interest. The experimenters feel further investigation and explanation is not warranted at this time but cautions APACTS developers of the anomaly's presence. As a precaution, the control system should execute an orderly shutdown if the feed pressure falls.

\section{References}

[1] Carderock Division, Naval Surface Warfare Center, SOL N00167-97-SS-R1, "Mechanical Ship Hull Paint Application System For Use in Drydock", Commerce Business Daily, April 10, 1997.

[2] R. Norcross, "One Degree Micro-Macro-manipulator Integration Test", NIST Technical Report 6562, Gaithersburg, MD 2000. 